\title{
A Facile Synthesis and Characterization of Biologically Active Halogen Substituted 1-Acetyl 3, 5-Diphenylpyrazole Derivatives
}

\author{
M Ganapathi $^{1}$, D Jayaseelan ${ }^{2}$ and S Guhanathan ${ }^{2 *}$ \\ ${ }^{1} P G$ \& Research Department of Chemistry, Government Arts College, Tiruvannamalai, Tamil Nadu, India \\ ${ }^{2} P G$ \& Research Department of Chemistry, Muthurangam Government Arts College (Autonomous), Vellore, Tamil Nadu, India
}

Received: September 05, 2015; Accepted: September 25, 2015; Published: October 15, 2015

*Corresponding author: S Guhanathan, PG \& Research Department of Chemistry, Muthurangam Government Arts College (Autonomous), Vellore, Tamil Nadu, India, E-mail: sai_gugan@yahoo.com

\begin{abstract}
A convenient and efficient method of halogen substituted 1-acetyl-3,5-diphenyl pyrazole derivatives were synthesized by the following two steps. In a first step, three halogen substituted acetophenones condensed with 3-hydroxy benzaldehydes through claisen-schmidt condensation reaction and which can be achieved by two solvents like ethanol and PEG-600. In a second step, the halogen substituted chalcones were condensed with hydrazine hydrate with acetic acid in the presence of piperidine as catalyst. The six synthesized compound structures were identified and confirmed by UV-Visible, FT-IR and ${ }^{1} \mathrm{H}$ NMR and Mass spectral analysis respectively. The anti-bacterial activities of compounds have also been tested using Minimum Inhibitory Concentration (MIC) method with two different microorganisms like Staphylococcus aureus (MTCC3381) and Escherichia coli (MTCC739). The results of the studies infer that the synthesised compounds found to have excellent antibacterial activity against the selected pathogenic organisms.
\end{abstract}

Keywords: Halogen Substituted Chalcones; PEG-600; Hydrazine Hydrate; Piperidine

\section{Introduction}

Flavonoids comprise a large family of plant derived polyphenolic compounds classified as flavonols, chalcones, aurones, flavanones, isoflavones, flavans, flavanonols, flavanols, and flavones differencing from each other in their structural group arrangements [1]. $\alpha, \beta$-Unsaturated ketones display a wide range of pharmacological properties, including cytotoxicity towards different cancer cell lines [2,3], antibiotic [4], antibacterial [5], antiviral [6], anti-inflammatory [7] and hepatoprotective activities [8]. They are well known intermediates for synthesizing various heterocyclic compounds like pyrazoline and pyrimidine derivatives. A survey of literature in the recent past reveals that some pyrazole derivatives possess antibacterial [9], antiinflammatory [10] and antifungal effects [11]. In the view of the above mentioned facts and our continued interest in the synthesis of heterocyclic compounds derived from chalcone precursors $[12,13]$, it was thought of interest to synthesize some new heterocyclic compounds containing pyrazole rings [14,15] and examine their antimicrobial properties.

Based on the careful analysis of the literature, in our present work a series of chalcones and 1-acetyl substituted pyrazoles compounds were synthesized. The synthesized compounds were characterized on the basis of UV-Visible, FTIR, ${ }^{1} \mathrm{H}$ NMR and mass spectral data. All the compounds were screened for their in vitro antibacterial activity against Gram positive strains (Staphylococcus aureus) and Gram negative strains (Escherichia coli) respectively.

\section{Experimental}

\section{Methods and materials}

The chemicals 3-hydroxybenzaldehyde 4-flouroacetophenone (2a), 4-chloroacetophenone 4-bromoacetophenone (2c), hydrazine hydrate (3), acetic acid, PEG-600, sodium hydroxide and piperidine were obtained from Sigma Aldrich and were used as such without further purification. Silica gel (TLC and Column grade) were purchased from Merck. The solvents were purified as per the standard procedure.

FTIR spectra (KBr pellets) were measured using Alpha Bruker FTIR instrument scanning with the entire region of $4000-400$ $\mathrm{cm}^{-1}$ with typical resolution of $1.0 \mathrm{~cm}^{-1}$. UV-Visible spectra were also recorder using Alpha Bruker UV spectrophotometer. The NMR spectra of the compounds have been recorded on Bruker AV400 spectrometer operating at $400 \mathrm{MHz}$ for recording ${ }^{1} \mathrm{H}$ NMR spectra in DMSO solvent using TMS as internal standard. Mass spectra have been recorded on SHIMADZU spectrometer using chemical ionization technique. Melting points of all synthesized compounds have been determined in open glass capillaries on Mettler FP51 melting point apparatus and are uncorrected.

\section{Synthesis}

The substituted chalcones were synthesised by two methods by using ethyl alcohol and PEG-600 as solvents. 


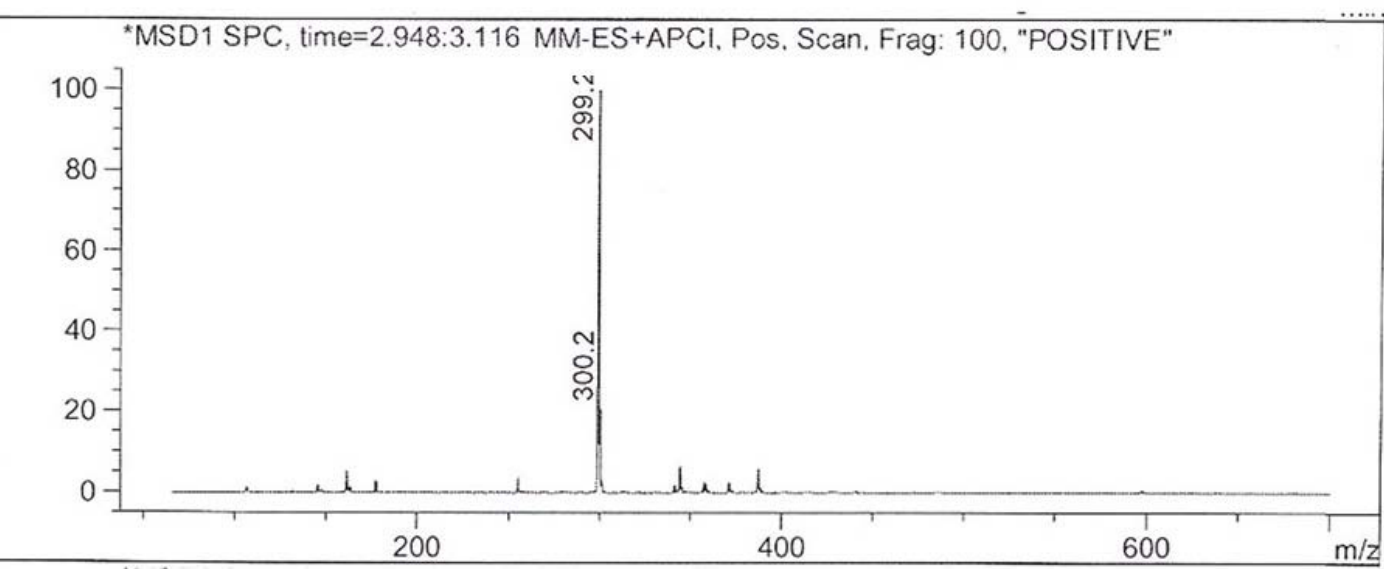

Figure 4: Mass Spectrum of the Compound - 5a.

Step - 1: General procedure for synthesis of compounds $4 a-4 c$

Method - A: To a stirred solution of $\mathbf{1}(0.0409 \mathrm{~mol})$ and 2a $2 c(0.0409 \mathrm{~mol})$ in ethanol $(50 \mathrm{~mL})$ cooled to $0^{\circ} \mathrm{C}$. To this sodium hydroxide solution $(3.27 \mathrm{~g}$ in $20 \mathrm{~mL}, 0.0818 \mathrm{~mol}$ ) were added drop wise over a period of 20 minutes. The reaction mixture was stirred at same temperature for 4-5 hours. Then the reaction mixture was kept in a refrigerator for overnight. The reaction was monitored by TLC which shows completion of reaction. The chilled reaction mixture warmed to room temperature, neutralised by using $\mathrm{HCl}(\mathrm{pH}=2-3)$, thrown out solid was stirred for a while and filtered, dried under vacuum. The purity of the product was checked by TLC (7:3, Hexane: Ethyl acetate as developing solvent) the solid obtained was recrystallized in methanol to yielded $\mathbf{4 a - 4 c .}$

Method - B: To a stirred suspension of $1(0.0409 \mathrm{~mol}), \mathbf{2 a}-$ 2c $(0.0409 \mathrm{~mol})$ and sodium hydroxide solution $(3.27 \mathrm{~g}$ in $20 \mathrm{~mL}$, $0.0818 \mathrm{~mol})$ in PEG-600 (20 mL) heated to $65^{\circ} \mathrm{C}$ for $1-2 \mathrm{~h}$. The completion of the reaction was monitored by TLC which shows consumption of starting material. The reaction mixture was cooled to $0^{\circ} \mathrm{C}$, neutralised using $\mathrm{HCl}(\mathrm{pH}=2-3)$, thrown out solid was filtered and dried under vacuum. The purity of the product was checked by TLC (7:3, Hexane: Ethyl acetate as developing solvent) the solid obtained was recrystallized in methanol, yielded 4a-4c. Filtrate was concentrated to remove water leaving behind PEG-600. The recovered PEG-600 has been reused for further synthesis of chalcones.

\section{Step- 2: General procedure for synthesis of compounds $5 a-5 c$}

To a stirred mixture of $\mathbf{4 a - 4 c}(0.0133 \mathrm{~mol})$ in acetic acid (10 V), hydrazine hydrate $(1.3 \mathrm{~mL}, 0.025 \mathrm{~mol})$ and piperidine $(2-3$ drops) were added. The resulting reaction mixture was refluxed for 2-3 hours. The completion of reaction was monitored by TLC. The reaction mixture was poured into ice-cold water. The thrown out solid was filtered and dried under vacuum. The solid obtained was recrystallized in ethyl acetate; afford $(\mathbf{5 a - 5 c})$.

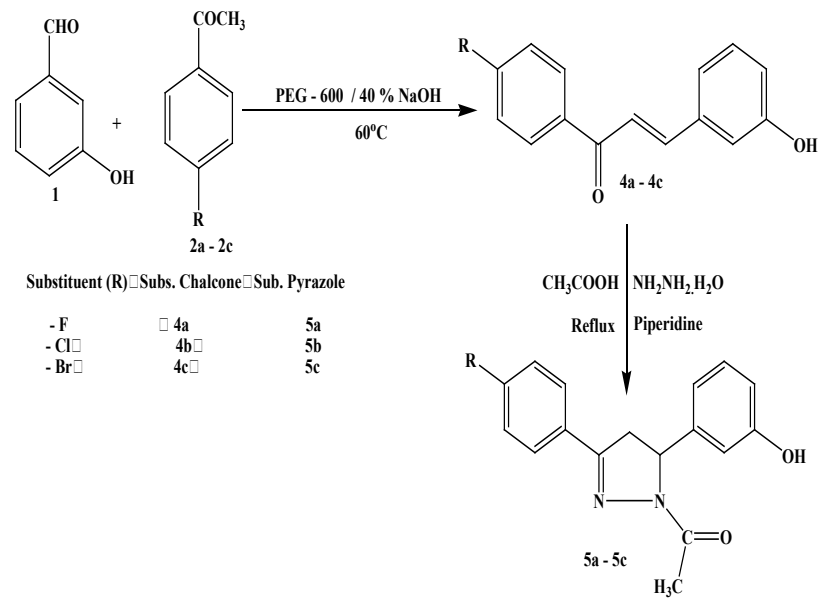

Scheme - 1: Synthesis of substituted 1-acetyl 3,5-diphenylpyrazoles.

\section{Results and Discussion}

Spectral details of 1-(4-fluorophenyl)-3-(3hydroxyphenyl)prop-2-en-1-one(4a)

Melting Point : $140-141^{\circ} \mathrm{C}$

Yield \& $\mathrm{R}_{\mathrm{f}}$ value : $94 \% \& 0.4324$

UV -Visible $\left(\lambda_{\max }: \mathrm{nm}\right): 226$ ( $\pi \rightarrow \pi^{*}$ transition), $314\left(\mathrm{n} \rightarrow \pi^{*}\right.$ transition) 